\title{
Unraveling the nucleation and growth of spontaneous surface relief gratings
}

\author{
Leila Mazaheri, ${ }^{a}$ Ribal Georges Sabat, ${ }^{\mathrm{b}}$ Olivier Lebel,,${ }^{\mathrm{c},}{ }^{,}$and, Jean-Michel Nunzi ${ }^{\mathrm{a}, \mathrm{d}, *}$ \\ ${ }^{a}$ Department of Physics, Queen's University, Kingston, ON, K7L 3N6, Canada \\ ${ }^{b}$ Department of Physics, Royal Military College of Canada, Kingston, ON, K7K 7B4, Canada \\ ${ }^{c}$ Department of Chemistry and Chemical Engineering, Royal Military College of Canada, Kingston, ON, K7K 7B4, Canada \\ ${ }^{d}$ Department of Chemistry, Queen's University, Kingston, ON, K7L 3N6, Canada \\ *Corresponding Author. E-mail address: nunzijm@queensu.ca (J.-M. Nunzi); Olivier.Lebel@,rmc.ca (O. Lebel).
}

\begin{abstract}
Nucleation and growth of spontaneous surface relief gratings (SSRGs) on a Disperse Red 1 (DR1) glass-forming derivative were investigated. No interference pattern is applied and surface patterning is induced using single-beam irradiation: the gratings are selforganized. Grating growth is assumed to initiate from an interference pattern formed between the incident light beam and waves scattered at grazing angle by surface defects. However, the mechanism is not yet fully understood and there is not a comprehensive explanation of the structure formation process. Herein, the grating formation procedure is studied by monitoring the surface topology of thin films exposed to one writing beam for various periods of time, under both linear and circular polarizations, using AFM. Even in the absence of surface defects on the initial film, irradiation produces light-induced surface defects due to the reorientation and mass movement of the azo molecules. These defects act as seeds for SSRG around which gratings gradually emerge and propagate throughout the sample. To consolidate this hypothesis, the formation of gratings was studied on samples with controlled surface roughness. Pore-shaped defects do not diffract light on top of the sample, and thus have no impact on SSRG growth, while for hill-shaped defects, growth rate decreases sharply with defect sizes larger than the writing beam wavelength. Two other analogous glass-forming azobenzene derivatives were studied, and in all cases, SSRG formation was correlated with the induction of birefringence in the early stages of the irradiation.
\end{abstract}

Keywords: $\quad$ azobenzenes, $\quad$ surface $\quad$ relief gratings, self-assembled, molecular glasses the driving force responsible for the macroscopic mass

\section{Introduction}

All-optical surface patterning of azobenzene derivatives has attracted a lot of attention in the literature in the past decade, both at the fundamental and applied levels. Materials containing azobenzene chromophores exhibit a range of photoresponsive properties, which all originate from their reversible photochemical cis-trans isomerization. ${ }^{1}$ While the photo-reorientation of azobenzene moieties induces birefringence, repeated cis-trans isomerization cycles caused by extended exposure to light results in mass movement in the material, eventually leading to macroscopic surface deformations. For example, irradiating a thin film of azobenzene-containing material with two laser beams forming an interference pattern induces a surface deformation where parallel grooves with well-defined depth and width form on the surface. While these surface relief gratings (SRG) have been widely studied, the fundamentals of their formation are not yet completely understood. It is well-known that SRG formation strongly depends on both intensity and polarization gradient, ${ }^{2}$ and more recently it has been shown that the surface deformation also depends on the phase and wavefront of the inscribing beams. ${ }^{3}$ Photo-fluidity due to isomerisation ${ }^{4}$ and $\pi$-shift of SRG relative to the interference pattern ${ }^{5}$ under moderate power irradiation (a few hundred $\mathrm{mW} / \mathrm{cm}^{2}$ ) are some of the main observed features underlying light-driven mass transfer. Several models have been proposed to explain transport of azo moieties on the micrometer scale at temperatures well below the glass transition temperature. For example, the pressure model, ${ }^{6}$ one of the first proposed models, attributes SRG formation to the change in volume occupied with azo isomers during isomerization. The mean field theory ${ }^{7}$ model proposed that SRG formation was due to the anisotropy of molecular interactions. The field gradient force model $^{8}$ postulates that SRG originate from a dependence towards the polarization and intensity gradients, in a fashion similar to optical tweezers. The diffusion model ${ }^{9}$ attributes SRGs to an inchworm-like motion of the azo moieties during cis-trans isomerization and reorientation. The random walk model, ${ }^{10}$ which is strictly equivalent to the latter model, confirmed the perspective of anisotropic light-driven molecular diffusion. Each model explains distinct aspects of SRG formation, but owing to the particular nature of the materials involved, none of them could provide a fully comprehensive explanation of all observed aspects of SRG formation.

More recently, it was demonstrated that SRG can be inscribed under uniform single-beam irradiation. ${ }^{11,12}$ No external interference pattern is applied, and thus the structures form spontaneously. A common proposed approach to these spontaneous surface relief gratings (SSRG) formation is the stimulated Wood anomaly model, ${ }^{13}$ in which a diffraction order is coupled into the sample. The incident beam is then scattered by tiny surface defects, leading to interferences 
between the scattered beams at the grazing angle and the incident beam. This self-organized interference pattern then initiates SSRG formation. As the grating grows, more light is trapped into the sample and couples into counter-propagating guided modes, which interfere with the incident beam, thereby causing the SSRGs to grow over the irradiated area. ${ }^{14}$ Molecular architecture has a predominant impact on reorientation and mass movement of an azo compound. Typically, SSRG growth is reported on azo polymers in which chain length and the nature of binding would determine the shape and the growth rate of SSRG. ${ }^{15-17}$ To mitigate the impact of the rheological effects of polymers, small molecules are an appealing alternative. ${ }^{18}$ Furthermore, small molecules yield a more homogeneous behavior, not only between different samples, but also within a given sample because all molecules are identical. ${ }^{19}$ While most azobenzene derivatives readily crystallize, thereby limiting their usefulness for thin film applications, a glass-forming material incorporating the Disperse Red 1 chromophore (thereafter referred to as DR1-glass) previously synthesized in our group has been shown to form high-quality amorphous thin films. ${ }^{19}$

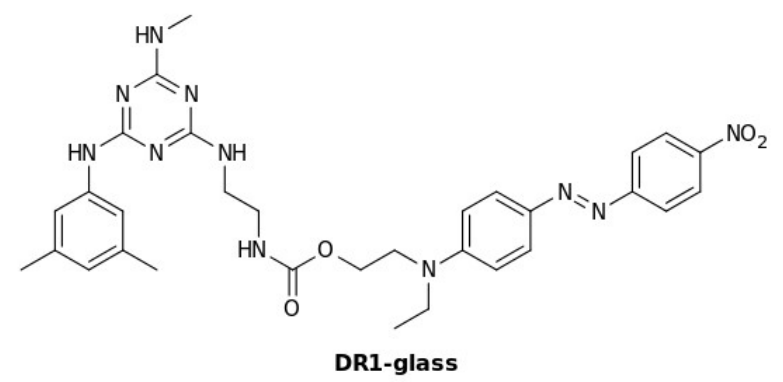

In an earlier study, growth and erasure of SSRGs on DR1-glass were investigated.20 It was proven that SSRG formation in azo-materials is exclusively an optical process and light polarization was the main factor determining the orientation of the structures. Under linear polarization, SSRGs form along two directions over a patchwork of domains, while circular polarization yields a circular crossed grating. SSRG formation follows hole burning saturation effect. The rate of grating growth increases as a function of laser intensity, but the gratings saturate at the same modulation amplitude independently of the laser intensity. Entanglement of light coupling in the SSRG growth process was confirmed by observing directional back-scattered light. It has been evidenced that SSRG grow due to interferences between the incident light and beams scattered by the sample. Nevertheless, our comprehension of how SSRG originate from surface defects, grow, and organize into well-defined domains remains inadequate.

SSRG formation takes place in three time-periods: nucleation, growth and saturation. Growth and saturation periods were previously modeled comprehensively using diffusion and coupled wave theory. ${ }^{14}$ Herein, the nucleation and growth of SSRG on films of DR1-glass is studied using Atomic Force
Microscopy (AFM). Samples with controlled surface roughness were prepared either using electric field corona poling on smooth thin films or during film deposition using various solvents, with the expectation that SSRG would grow at an even greater rate in these cases, because of the light scattering caused by the already present defects. ${ }^{21}$ Birefringence was monitored in DR1-glass and two analogues with similar chromophores to establish a correlation between SSRG nucleation and the induction of birefringence in the early stages of irradiation. Our findings have allowed to clarify the mechanism by which SSRG nucleate and grow from irradiation with a single beam.

\section{Experimental}

\subsection{Thin Film Deposition}

Smooth thin films of DR1-glass was prepared on glass substrates by spin-coating from a $3 \mathrm{wt} \% \mathrm{CH}_{2} \mathrm{Cl}_{2}$ solution on BK7 glass substrates $\left(3 \times 3 \mathrm{~cm}^{2}\right)$. The solution was submitted to mechanical shaking for 1 hour, and then filtered through a $0.45 \mu \mathrm{m}$ filter. The deposited thin films were then dried at 95 ${ }^{\circ} \mathrm{C}$ for 5 minutes to remove any traces of solvent. This procedure yielded films with a thickness of approximately $270 \mathrm{~nm}$, and an absorbance of 1.125 at the working wavelength $(532 \mathrm{~nm})$ and 0.01 at the probe beam wavelength $(633 \mathrm{~nm})$. Dewetted films containing dome patterns could be obtained by spin-coating from $\mathrm{CH}_{2} \mathrm{Cl}_{2}$ /ethanol (9:1) solution under the same conditions, while films with pores could be generated by spin-coating from $\mathrm{THF} / \mathrm{H}_{2} \mathrm{O}(9: 1){ }^{22}$

\subsection{Electric Field Poling}

The surface roughness of DR1-glass films could be increased by using corona poling using a process described in the literature. ${ }^{23}$ Samples were annealed at various temperatures above their glass transition temperatures $\left(\mathrm{T}_{\mathrm{g}}\right)$ while an electric field was applied. Temperature was controlled using ColeParmer Digi-Sense temperature controller and a hot plate. Samples were placed on a hot plate and heated to the desired temperature $\left(75\right.$ or $\left.90{ }^{\circ} \mathrm{C}\right)$, then an electric field of $8 \mathrm{kV}$ was applied for $30 \mathrm{~min}$. The metallic hot plate surface acts as one of the electrodes, while the other electrode was a thin metallic wire, which was held horizontally $0.9 \mathrm{~cm}$ above the sample. The corona discharge voltage was supplied by a Hippotronics High-Voltage DC power supply. Samples with root mean square (RMS) surface roughness ranging from roughly 5 to $125 \mathrm{~nm}$, as measured by AFM, could be generated depending on the temperature.

\subsection{Surface Relief Grating Inscription}

The setup used to inscribe SRG is identical to that described in the literature. ${ }^{20} \mathrm{~A} \mathrm{Nd}$-YAG laser $(\lambda=532 \mathrm{~nm})$ was used as the pump beam. The size of the collimated laser beam impinging onto the azo test sample was controlled with a Keplerian beam expander system. The sample was set perpendicular to the incident laser beam, while the laser beam 
polarization was set vertically. The polarization state can be varied from linear to elliptical using half-wave and quarter wave plates. Formation of spontaneous SRG was monitored by atomic force microscopy (AFM).

\subsection{Atomic Force Microscopy}

The morphology of the samples was studied using an Ambios Technology Q-Scope TM 250/400 Nomad Scanning Probe Microscope, operated in wave mode using BS-tap 300 Al tips (NANOANDMORE). The AFM scans were analyzed using the Gwyddion software package. ${ }^{24}$

\subsection{Birefringence Measurement}

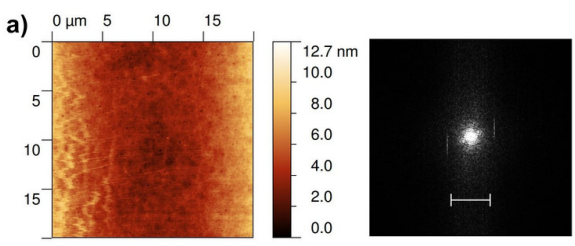

$$
\text { b) } 0.0 \mu \mathrm{m} \quad 5.0
$$
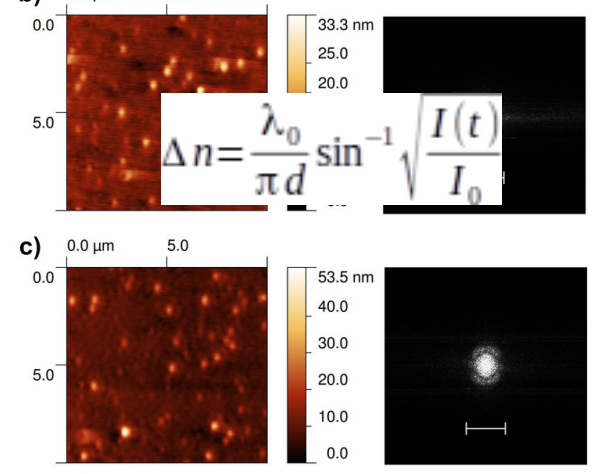

$$
\text { d) } 0.0 \mu \mathrm{m} \quad 5.0
$$

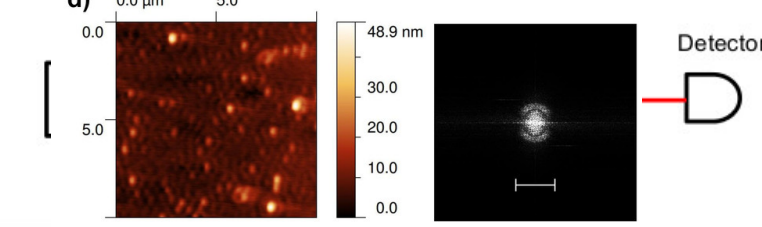

\begin{tabular}{l}
\hline Pur \\
532 \\
\hline
\end{tabular}
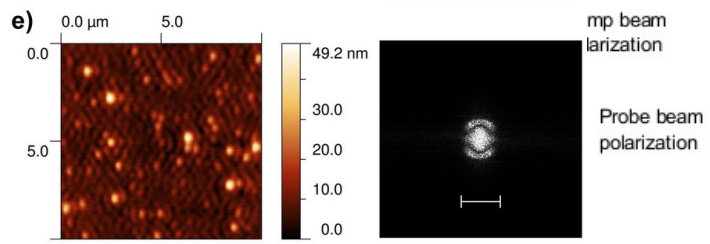

f) $0.0 \mu \mathrm{m} \quad 5.0$

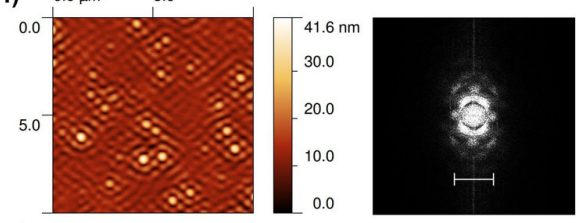

g) $0.0 \mu \mathrm{m} \quad 5.0$

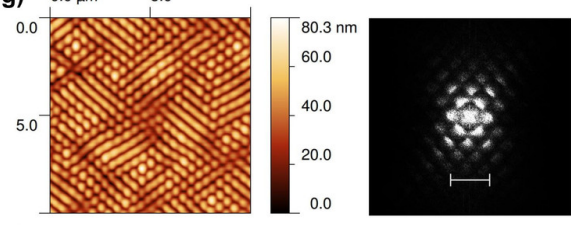

h) $0.0 \mu \mathrm{m} \quad 5.0$

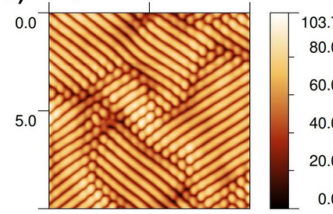

Photoinduced birefringence was monitored using a $\mathrm{He}-\mathrm{Ne}$ laser with an irradiance of $80 \mathrm{~mW} / \mathrm{cm}^{2}$. The sample was placed between crossed polarizers, and the transmission of the probe beam was recorded. An Nd-YAG laser with a wavelength of $532 \mathrm{~nm}$ and an irradiance of $65 \mathrm{~mW} / \mathrm{cm}^{2}$ was incident at 45-degrees onto the azo film, as shown in Figure 1. Photo-isomerization and reorientation of the azo molecules induces photo-anisotropy, and the change in refractive index is calculated by the equation:

Where $\lambda_{0}, d, I(t)$ and $I_{0}$ are the wavelength of the probe beam, the thickness of the film, and the irradiances of the transmitted and incident probe beams, respectively. The pump beam power was kept low to induce birefringence without causing surface deformation.

Figure 1. Birefringence measurement setup.

\section{Results and Discussion}

The SSRG growth process was studied by monitoring the surface topology by AFM of DR1-glass films exposed to the Nd-YAG writing beam for various periods of time under linear polarization (Figure 2). The surface of the film was first confirmed to be topologically flat. Irradiation with the writing beam initially induces deformations on the surface of the material in the form of circular protrusions approximately 500 Figure 2. AFM images of films of DR1-glass irradiated with a linearly polarized laser with $\lambda=532 \mathrm{~nm}$ and an irradiance of $300 \mathrm{~mW} / \mathrm{cm}^{2}$ for various periods of time: (a) $0 \mathrm{~s}$, (b) $30 \mathrm{~s}$, (c) $45 \mathrm{~s}$, (d) $60 \mathrm{~s}$, (e) $90 \mathrm{~s}$, (f) $5 \mathrm{~min}$, (g) $10 \mathrm{~min}$, and (h) $15 \mathrm{~min}$. Two-dimensional Fourier transforms are also shown for each image. The laser polarization direction is set vertical with respect to the figure.

$\mathrm{nm}$ in diameter and with height increasing as a function of irradiation time (Figure 2b-c). These light-induced defects then nucleate SSRG by scattering the incident light, thereby generating an interference pattern between the incident and scattered beams, leading to the formation of ripples in a rhombic lattice around these defects (Figure 2d-e). Those ripples then gradually spread over the surface, and gradually deepen to generate a patchwork of domains along two different orientations, with some areas still part of two crossed gratings (Figure 2f-g). These domains eventually equilibrate into larger domains with distinct boundaries (Figure 2h). Two-dimensional Fourier transforms of these AFM images illustrate the growth process of these gratings, first with the formation of reflections in a rhombic pattern, then with the apparition of longer range reflections along two orientations.

Although about twice as fast, the same process is observed under circular polarization (Figure 3). Circular protrusions about $500 \mathrm{~nm}$ in diameter first form, then ripples in a circular pattern emerge from the material around these protrusions, 
eventually leading to the formation of a circular grating where the surface is littered with similarly shaped grains arranged in hexagonal patterns, rather than parallel grooves.

Spontaneous surface relief gratings (SSRGs) thus initiate from surface defects induced by light due to the reorientation and mass movement of azo molecules. These irregularities then scatter a small amount of light out of the primary laser beam. The incident beam then forms an interference pattern between the incident beam and the waves scattered at grazing incidence, which in turn induces an ordered surface pattern. Not only do these observations further confirm the model by which light coupling caused by surface defects is responsible for SSRG formation, but the observation of surface deformations induced by the incident beam also helps rationalize how SSRG form and propagate rapidly on topologically flat surfaces.

By graphically removing the gratings from the images, it is possible to observe the topology of the underlying film (Figure 4). While the film is initially smooth, SSRG formation under linear polarization induces the formation of plateaus in the film separated by ridges and crevices (Figure 4a-b). On the other hand, irradiation under circular polarization induces some degree of deformation, but mostly in the form of circular holes (Figure 4c-d). Upon closer observation, the different plateaus observed in SSRGs grown under linear polarization correspond to the different SSRG domains in the films, while the ridges and valleys are located at the boundaries between domains. It is believed that these surface irregularities emerge from molecular diffusion due to domain growth, which exerts either a converging (ridges) or

a) $0 \mu \mathrm{m} \quad 5 \quad 10 \quad 15$

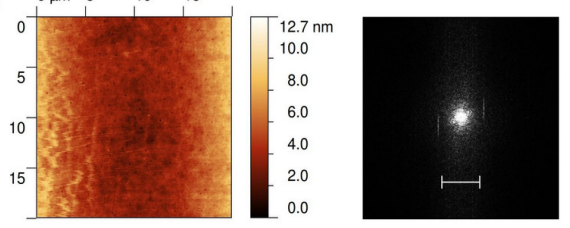

b) $0.0 \mu \mathrm{m} \quad 5.0$

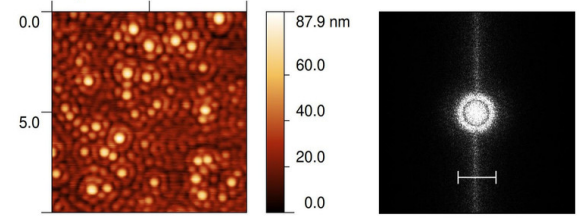

c) $0.0 \mu \mathrm{m} \quad 5.0$

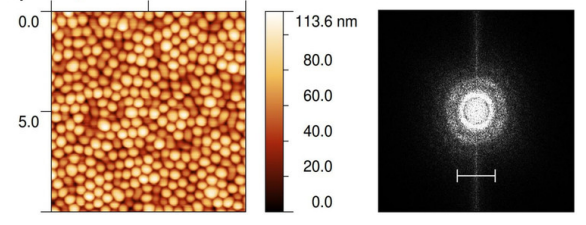

d) $0.0 \mu \mathrm{m} \quad 5.0$

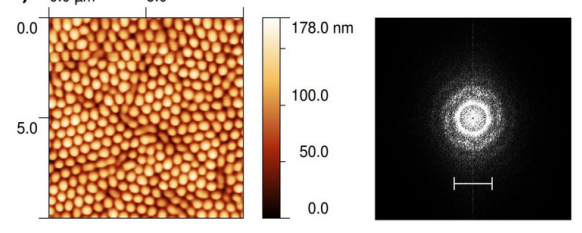

e) $0.0 \mu \mathrm{m} \quad 5.0$

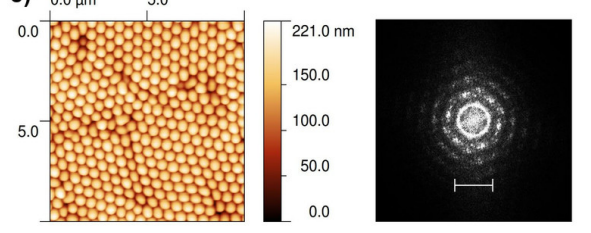

diverging (valleys) force at the boundaries. On the other hand, as circular SSRGs do not form separate domains with specific directionality, no such deformations are observed.

Figure 3. AFM images of films of DR1-glass irradiated with a circularly polarized laser with $\lambda=532 \mathrm{~nm}$ and an irradiance of $300 \mathrm{~mW} / \mathrm{cm}^{2}$ for various periods of time: (a) $0 \mathrm{~s}$, (b) $150 \mathrm{~s}$, (c) $5 \mathrm{~min}$, (d) $10 \mathrm{~min}$, (e) $60 \mathrm{~min}$. Two-dimensional Fourier transforms are also shown for each image.

As SSRGs originate from surface defects that are induced by light during the initial phase of irradiation, SSRG growth was studied on films of DR1-glass in which surface defects were previously generated, with the expectation that SSRGs would grow at an even greater rate in these cases, because of the light scattering caused by the already present defects. For this study, films with varying degrees of surface roughness and defect shapes were prepared according to four conditions: 1) electrical poling under a $8 \mathrm{kV}$ field at $75^{\circ} \mathrm{C}$ for 30 minutes, 2) electrical poling under a $8 \mathrm{kV}$ field at $85^{\circ} \mathrm{C}$ for 30 minutes, 3) deposition by spin-coating from $\mathrm{THF} / \mathrm{H}_{2} \mathrm{O}$ (9:1), and 4) deposition by spin-coating from $\mathrm{CH}_{2} \mathrm{Cl}_{2} / \mathrm{EtOH}$ (9:1). These procedures and the mechanisms accounting for their respective dewetting behavior have been reported in previous studies. $^{22,23}$ Initial average RMS surface roughness for films prepared under these conditions are listed in Table 1.

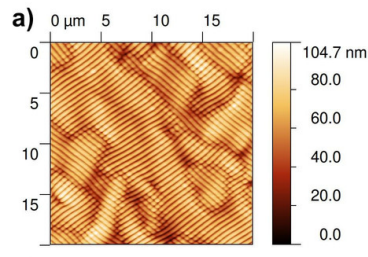

c) $0 \mu \mathrm{m} \quad 5 \quad 10 \quad 15$

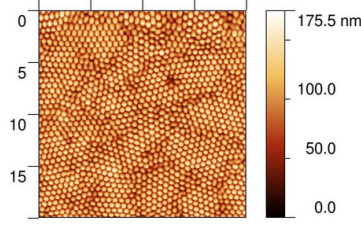

b) $0 \mu \mathrm{m} \quad 5 \quad 10 \quad 15$

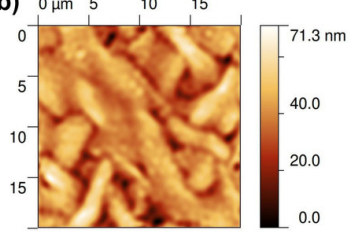

d) $0 \mu \mathrm{m} \quad 5 \quad 10 \quad 15$

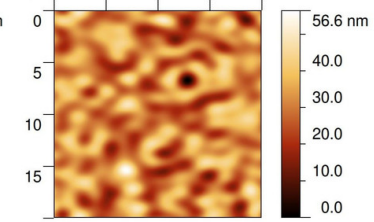

Figure 4. AFM images of films of DR1-glass irradiated with a laser with $\lambda=532 \mathrm{~nm}$ and an irradiance of $300 \mathrm{~mW} / \mathrm{cm}^{2}$ for 25 min: (a) linear polarization, (b) the image in (a) with the grating graphically removed using a two-dimensional Fourier transform filter, (c) circular polarization, (d) the image in (c) with the grating graphically removed using a two-dimensional Fourier transform filter.

Table 1. Initial RMS surface roughness for films of DR1glass.

\begin{tabular}{ll} 
Film Processing & RMS Surface Roughness (nm) \\
\hline Spin-coating, $\mathrm{CH}_{2} \mathrm{Cl}_{2}$ & 1.69 \\
$\begin{array}{l}\text { Electrical poling, } 8 \mathrm{kV}, 75^{\circ} \mathrm{C}, \\
\begin{array}{l}30 \text { min } \\
l\end{array}\end{array}$ & 8.6 \\
\end{tabular}


Electrical poling, $8 \mathrm{kV}, 85^{\circ} \mathrm{C}, \quad 128$

$30 \mathrm{~min}$

Spin-coating, $\mathrm{THF} / \mathrm{H}_{2} \mathrm{O}$

90.4

Spin-coating, $\mathrm{CH}_{2} \mathrm{Cl}_{2} / \mathrm{EtOH}$

144

DR1-glass samples poled at $75{ }^{\circ} \mathrm{C}$ for 30 minutes yielded films that contained a surface defect density similar to that observed during the initial irradiation with the writing beam. Expectedly, SSRG formed on these pre-patterned films at a rate similar to that observed for topologically flat films, for both linear (Figure 5) and circular polarization (Figure 6). Unexpectedly, while the SSRG depth increased at a similar rate, the domain organization progressed much slower in the case of linear polarization, with significant portions of material part of crossed gratings, even after 25 minutes. After extended exposure (90
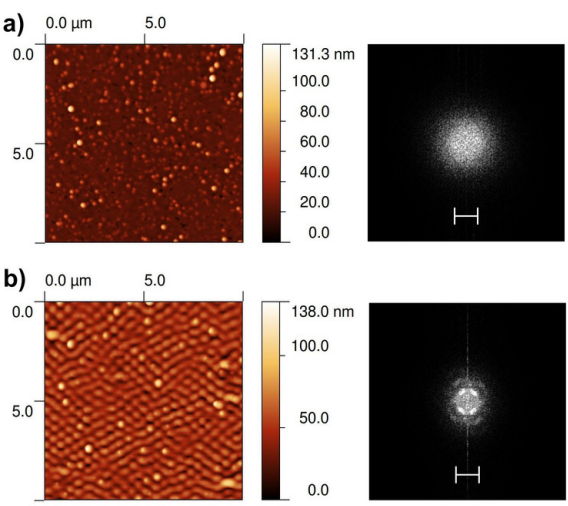

c) $0.0 \mu \mathrm{m} \quad 5.0$

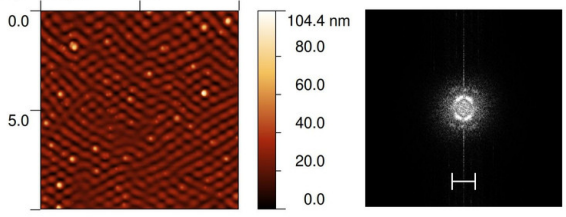

d) $0.0 \mu \mathrm{m} \quad 5.0$

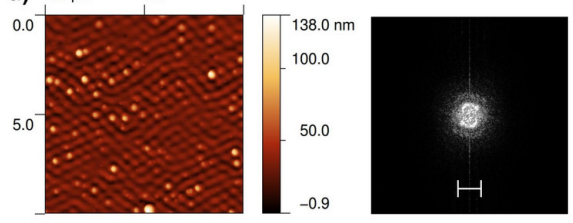

e) $0.0 \mu \mathrm{m} \quad 5.0$

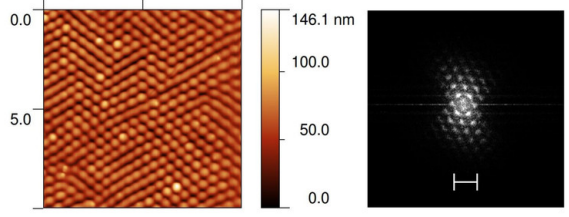

f) $0.0 \mu \mathrm{m} \quad 5.0$

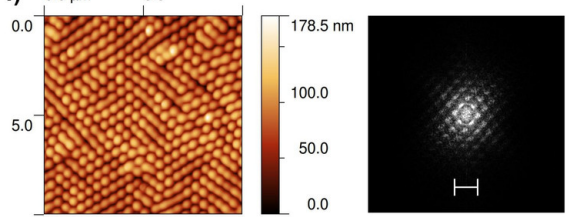

g) $0.0 \mu \mathrm{m} \quad 5.0$

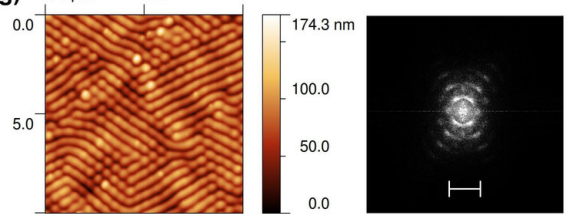

minutes), domains were mostly formed, but even in this case, the domains were much smaller than those of SSRG inscribed on smooth films. Counting the number of grains in a $100 \mu \mathrm{m}^{2}$ area has revealed an average of approximately 30 grains for smooth films after $30 \mathrm{~s}$ irradiation, in comparison to 150 grains for films dewetted at $75{ }^{\circ} \mathrm{C}$. Domain size thus seems to be correlated with the density of grains. As each grain nucleates gratings along two orientations, a higher grain density will result in more overlap between nascent gratings, which lead to increased competition and a higher area of crossed gratings, thereby reducing domain size.

Figure 5. AFM images of films of DR1-glass poled at $75^{\circ} \mathrm{C}$ under a $8 \mathrm{kV}$ field for $30 \mathrm{~min}$ and irradiated with a linearly polarized laser with $\lambda=532 \mathrm{~nm}$ and an irradiance of 300 $\mathrm{mW} / \mathrm{cm}^{2}$ for various periods of time: (a) $0 \mathrm{~s}$, (b) $150 \mathrm{~s}$, (c) 5 min, (d) $10 \mathrm{~min}$, (e) $15 \mathrm{~min}$, (f) $25 \mathrm{~min}$, and (g) $90 \mathrm{~min}$. Twodimensional Fourier transforms are also shown for each image. The laser polarization direction is set vertical with respect to the figure.
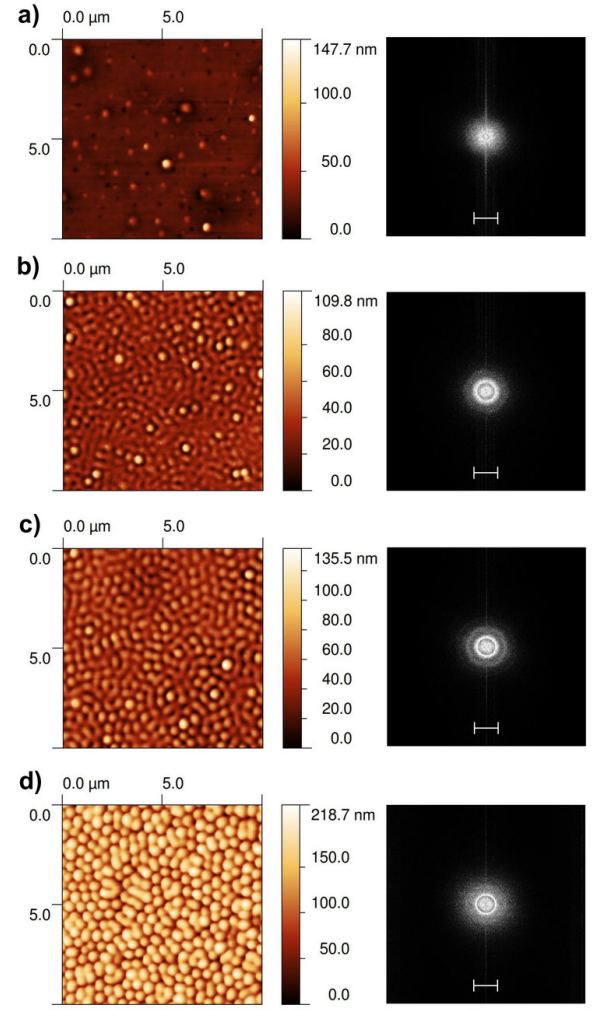

Figure 6. AFM images of films of DR1-glass poled at $75^{\circ} \mathrm{C}$ under a $8 \mathrm{kV}$ field for $30 \mathrm{~min}$ and irradiated with a circularly polarized laser with $\lambda=532 \mathrm{~nm}$ and an irradiance of 300 $\mathrm{mW} / \mathrm{cm}^{2}$ for various periods of time: (a) $0 \mathrm{~s}$, (b) $60 \mathrm{~s}$, (c) 150 $\mathrm{s}$, and (d) $10 \mathrm{~min}$. Two-dimensional Fourier transforms are also shown for each image.

Films spin-coated from a $\mathrm{THF} / \mathrm{H}_{2} \mathrm{O}(9: 1)$ mixture showed an 
irregular surface topology littered with pores rather than grains. These concave structures cannot scatter light in a way that can cause an interference pattern necessary for SSRG formation. It can be observed that unlike films with small grains, films with pores behaved similarly to topologically smooth films, with surface deformations occurring within 30 $\mathrm{s}$ of irradiation, then SSRG nucleating, growing, and organizing into domains (Figure 7). The presence of pores thus did not perturb the SSRG formation process, thereby implying that SSRG formation is a surface-initiated phenomenon, as these pores do not contribute to light diffraction inside the film.

Graphically filtering the gratings from the images of films poled electrically reveals an absence of boundary deformations, in sharp contrast with smooth films (Figure 8ab). This is arguably a result of smaller and less well-defined domains. This graphical treatment also enables to clearly observe the deformation of the pores in films spin-coated from $\mathrm{THF} / \mathrm{H}_{2} \mathrm{O}$ (Figure $8 \mathrm{c}-\mathrm{d}$ ). It can be observed that the formation of SSRG causes a gradual elongation of the initially circular pores along the direction of the polarization, which is caused by photofluidization in the direction parallel to the polarization direction.

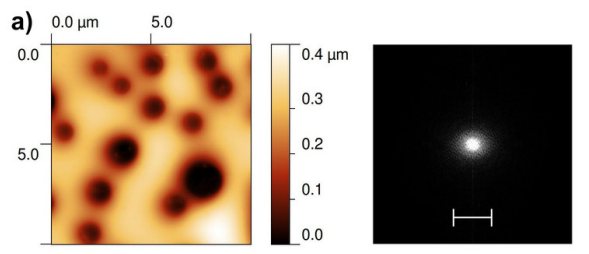

b) $0.0 \mu \mathrm{m} \quad 5.0$

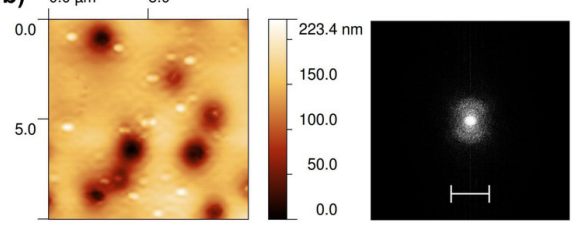

c) $0.0 \mu \mathrm{m} \quad 5.0$

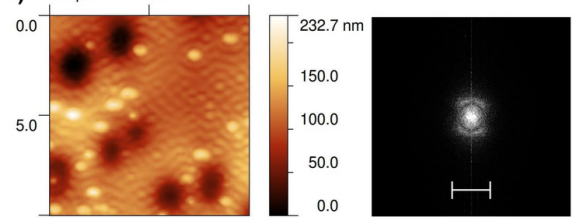

d) $0.0 \mu \mathrm{m} \quad 5.0$

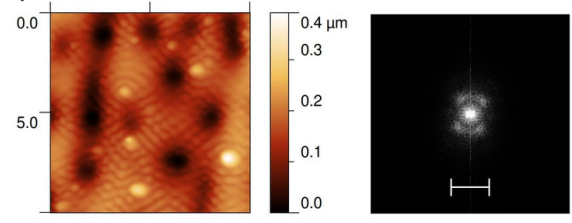

e) $0.0 \mu \mathrm{m} \quad 5.0$

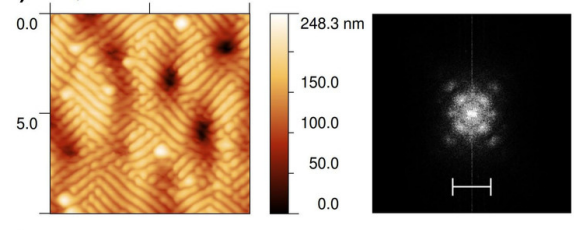

f) $0.0 \mu \mathrm{m} \quad 5.0$

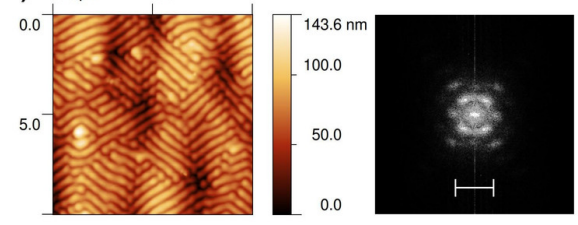

Figure 7. AFM images of films of DR1-glass spin-coated from $\mathrm{THF} / \mathrm{H}_{2} \mathrm{O}$ and irradiated with a linearly polarized laser with $\lambda=532 \mathrm{~nm}$ and an irradiance of $300 \mathrm{~mW} / \mathrm{cm}^{2}$ for various periods of time: (a) $0 \mathrm{~s}$, (b) $60 \mathrm{~s}$, (c) $150 \mathrm{~s}$, (d) $5 \mathrm{~min}$, (e) 10 min, and (f) $20 \mathrm{~min}$. Two-dimensional Fourier transforms are also shown for each image. The laser polarization direction is set vertical with respect to the figure.
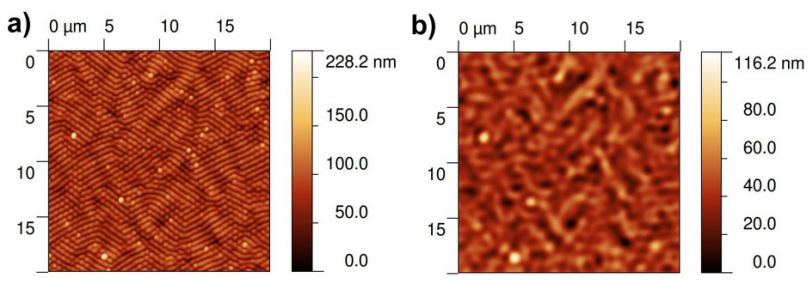

C) $0 \mu \mathrm{m} \quad 5 \quad 10 \quad 15$

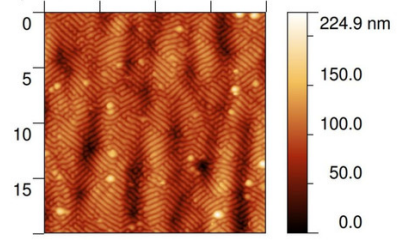

d) $0 \mu \mathrm{m} \quad 5 \quad 10 \quad 15$

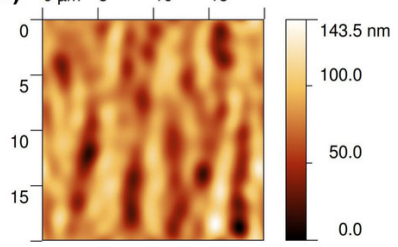

Figure 8. AFM images of films of DR1-glass irradiated with a laser with $\lambda=532 \mathrm{~nm}$ and an irradiance of $300 \mathrm{~mW} / \mathrm{cm}^{2}$ : (a) film of DR1-glass poled at $75{ }^{\circ} \mathrm{C}$ under a $8 \mathrm{kV}$ field for 30 min and irradiated for $90 \mathrm{~min}$, (b) the image in (a) with the grating graphically removed using a two-dimensional Fourier transform filter, (c) film of DR1-glass spin-coated from $\mathrm{THF} / \mathrm{H}_{2} \mathrm{O}$ and irradiated for $20 \mathrm{~min}$, (d) the image in (c) with the grating graphically removed using a two-dimensional Fourier transform filter.

In the case of films containing large grains, which were obtained by spin-coating from $\mathrm{CH}_{2} \mathrm{Cl}_{2} / \mathrm{EtOH}, \mathrm{SSRG}$ formed at a much slower rate, with small grooves being noticeable after 5 to 15 minutes exposure, and fully formed SSRG being visible after 30 minutes (Figure 9). A similar behavior was observed with films dewetted by electrical poling at $85^{\circ} \mathrm{C}$ (Figure 10a-e). In these cases, the directionality of the gratings is dependent on the shape of the grains and local curvature, with the direction of the grooves roughly perpendicular to the longest dimension of the grains. In a fashion similar to the elongation of the pores observed in the films deposited from $\mathrm{THF} / \mathrm{H}_{2} \mathrm{O}$, SSRG growth in these two cases caused the gradual flattening of the grains. Under linear polarization, this flattening was more accentuated in the direction perpendicular to the grating grooves. On the other hand, this effect was significantly more pronounced under circular polarization (Figure 10f-i), where after 1 hour exposure, the presence of a grainy surface had completely disappeared, with the resulting SSRG being similar to those inscribed on an untreated film. If photofluidization leads to deformation of the surface defects in the direction of the polarization, circular polarization leads to a simultaneous flattening in all the directions, leading to a faster smoothing 
of the surface.
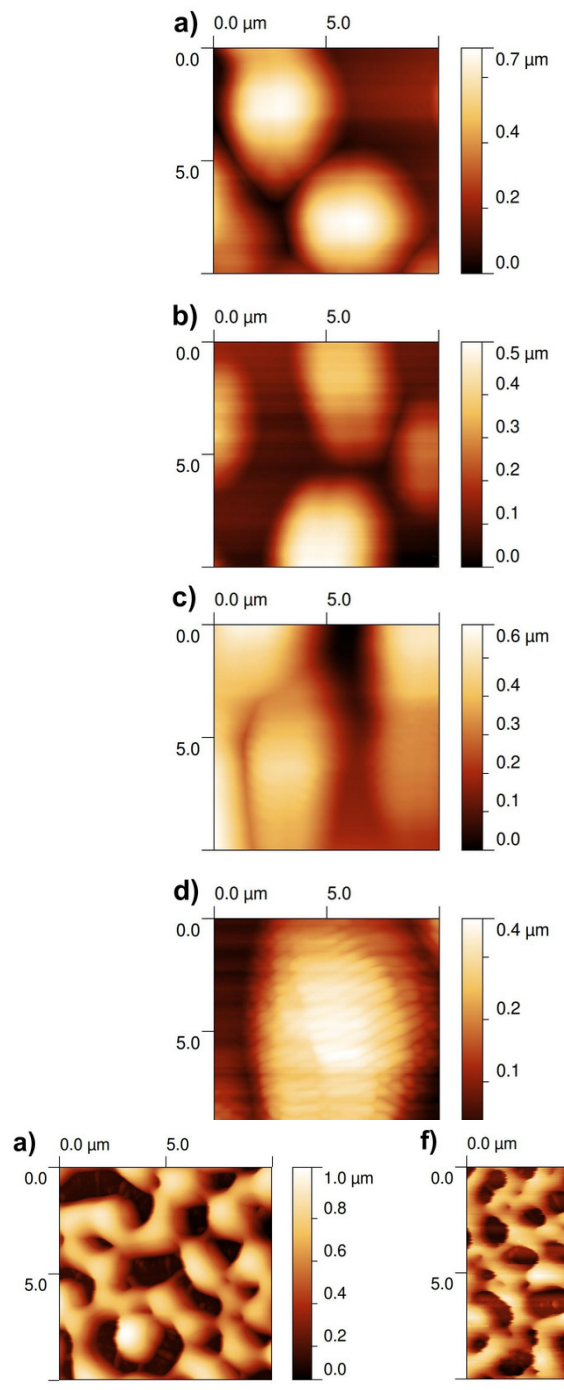

b) $0.0 \mu \mathrm{m} \quad 5.0$

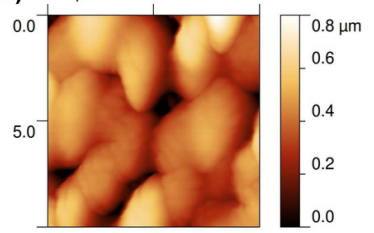

c) $0.0 \mu \mathrm{m} \quad 5.0$

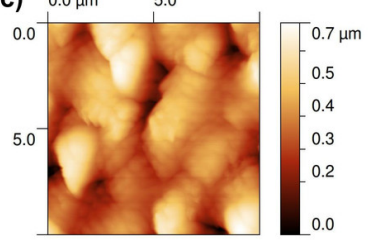

d) $0.0 \mu \mathrm{m} \quad 5.0$

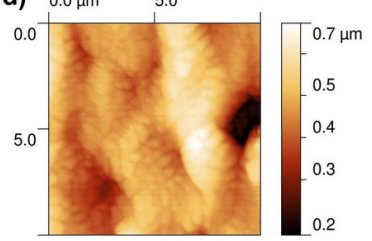

e) $0.0 \mu \mathrm{m} \quad 5.0$

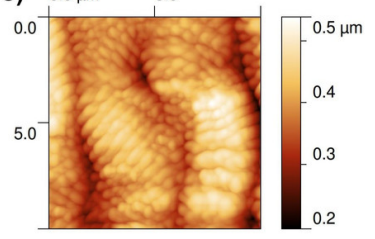

Figure 9. AFM images of films of DR1-glass spin-coated from $\mathrm{CH}_{2} \mathrm{Cl}_{2} / \mathrm{EtOH} \quad(9: 1)$ and irradiated with a linearly polarized laser with $\lambda=532 \mathrm{~nm}$ and an irradiance of 300 $\mathrm{mW} / \mathrm{cm}^{2}$ for various periods of time: (a) $0 \mathrm{~s}$, (b) $5 \mathrm{~min}$, (c) 15 min, (d) $30 \mathrm{~min}$, and (e) $60 \mathrm{~min}$. The laser polarization direction is set vertical with respect to the figure.

Figure 10. AFM images of films of DR1-glass poled at $85^{\circ} \mathrm{C}$ under a $8 \mathrm{kV}$ field for $30 \mathrm{~min}$ and irradiated with a linearly or circularly polarized laser with $\lambda=532 \mathrm{~nm}$ and an irradiance of $300 \mathrm{~mW} / \mathrm{cm}^{2}$ for various periods of time: (a-e) linear polarization: (a) $0 \mathrm{~s}$, (b) $5 \mathrm{~min}$, (c) $15 \mathrm{~min}$, (d) $30 \mathrm{~min}$, and (e) $60 \mathrm{~min}$; (f-i) circular polarization: (f) $0 \mathrm{~s}$, (g) $5 \mathrm{~min}$, (h) 15 min, (i) $60 \mathrm{~min}$. For linear polarization, the laser polarization direction is set vertical with respect to the figure.

SSRG formation was also probed for two analogous azobenzene glass-forming derivatives with 4(phenylazo)phenyl and 6-nitrobenzathiazolyl groups, termed Azoglass-470 and Azoglass-530, respectively. ${ }^{25}$ The $\lambda_{\max }$ values for films of both compounds are 470 and $538 \mathrm{~nm}$, respectively, and their respective molar absorptivity coeeficients at $532 \mathrm{~nm}\left(\varepsilon_{532}\right)$ are 12900 and $23800 \mathrm{M}^{-1} \mathrm{~cm}^{-1}$ (in comparison, E532 for DR1-glass is $13900 \mathrm{M}^{-1} \mathrm{~cm}^{-1}$ ). Azoglass470 is known to form SRG upon irradiation with interfering laser beams at a rate slightly slower than DR1-glass, while irradiation of Azoglass-530 only yields very shallow gratings, and requires a stronger irradiance and longer exposure times. Single-beam irradiation of Azoglass-470 films was found to result in surface deformations in the initial stages of exposure, followed by the formation of small SSRG following two domain orientations, in a fashion similar to DR1-glass (Figure 11a-e). However, in the case of Azoglass-470, the surface deformations generated were larger than for DR1-glass, and continued to grow with time, while the gratings obtained were significantly smaller, with a maximal amplitude of 20-30 nm reached after $5 \mathrm{~min}$, and a grating pitch similar to that of DR1-glass. On the other hand, irradiation of Azoglass-530 films under the same conditions did not result in any changes in the topology of the films (Figure 11f-h). No surface deformations were observed, even after 10 minutes exposure, and consequently, no SSRG were formed. These observations further confirm the role of these surface deformations formed in the initial stages of irradiation as seeds for the formation of SSRG. 

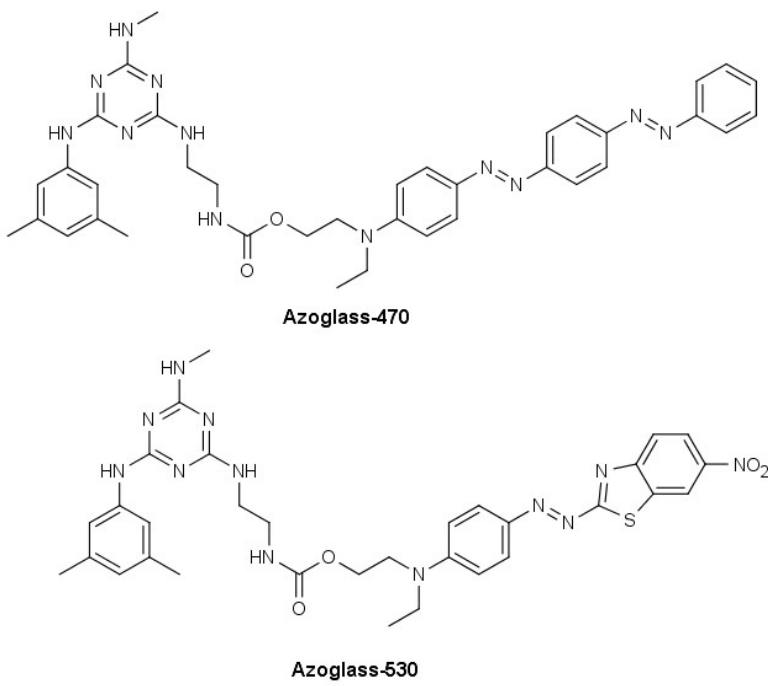

As birefringence is known to be induced in the first stages of irradiation of azobenzene-containing materials as a consequence of dipole alignment, the birefringence of films of DR1-glass, Azoglass-470 and Azoglass-530 was monitored during irradiation (Figure 12). While the rates of birefringence induction for DR1-glass and Azoglass-470 are closely similar, very little birefringence is observed for Azoglass-530 other than an initial rise attributed to rapid cistrans isomery, hinting that the alignment of the chromophores
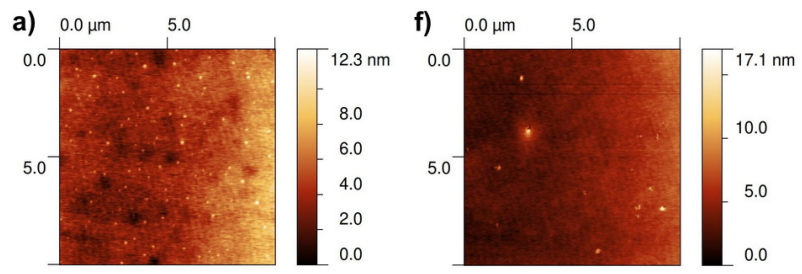

b) $0.0 \mu \mathrm{m} \quad 5.0$

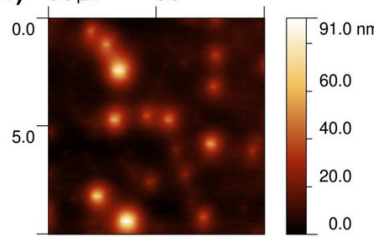

C) $0.0 \mu \mathrm{m} \quad 5.0$
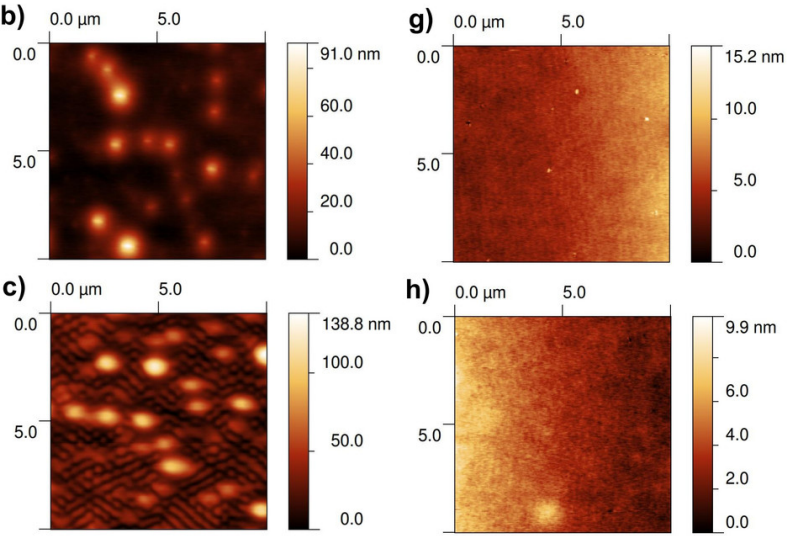

h) $0.0 \mu \mathrm{m} \quad 5.0$

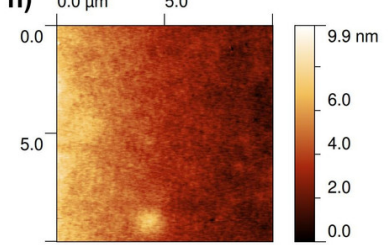

of Azoglass-530 is very slow, which prevents mass movement from occurring in the material at a rate necessary for SSRG formation. For DR1-glass and Azoglass-470, the similar rates of birefringence induction suggest that reorientation of azo moieties happens at the same rate. However, mass movement appears to be more chaotic in Azoglass-470, as evidenced by the formation of larger spherical defects and the lower maximal grating amplitude. This could be due to the presence of two azo groups in the chromophore of Azoglass-470, which can each isomerize separately and give rise to four different isomers. Arguably, the isomerization process in Azoglass-470 is fast but less controlled, resulting in rapid agglomeration of the molecules in surface defects, which do not scatter light efficiently in the sample surface, thereby leading to smaller gratings.

Figure 11. AFM images of films of Azoglass-470 and Azoglass-530 irradiated with a linearly polarized laser with $\lambda=532 \mathrm{~nm}$ and an irradiance of $300 \mathrm{~mW} / \mathrm{cm}^{2}$ for various periods of time. (a-e) Azoglass-470: (a) $0 \mathrm{~s}$, (b) $30 \mathrm{~s}$, (c) 5 min, (d) $10 \mathrm{~min}$, and (e) $20 \mathrm{~min}$; (f-h) Azoglass-530: (f) $0 \mathrm{~s}$, (g) $5 \mathrm{~min}$, and (h) $10 \mathrm{~min}$. The laser polarization direction is set vertical with respect to the figure.

With all these observations about initial protrusion formation leading to the efficient construction of well-defined SSRGs, it is legitimate to question about the origin of these hills. We can apply Hertz theory to find out the pressure $P$ that would be needed to induce the surface protrusions with $2 a=500 \mathrm{~nm}$ diameter and height $d=20 \mathrm{~nm}$ in Figure $2 \mathrm{~b} .^{26}$

In which $E \approx 4000 \mathrm{MPa}$ is the Young modulus of the glass measured by dynamic mechanical analysis ${ }^{27}$ and a Poisson ratio of 0.5 was assumed for an incompressible glass. We get $P \approx 180 \mathrm{MPa}$.

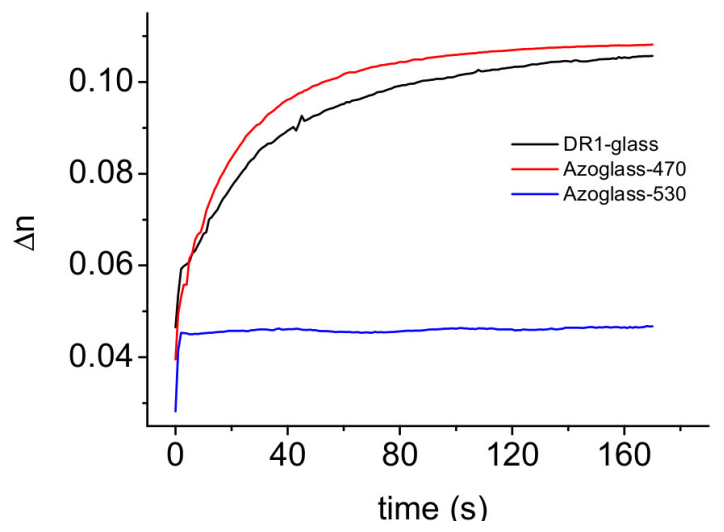

Figure 12. Photo-induced birefringence as a function of time for DR1-glass, Azoglass-470 and Azoglass-530. e) $0.0 \mu \mathrm{m} \quad 5.0$

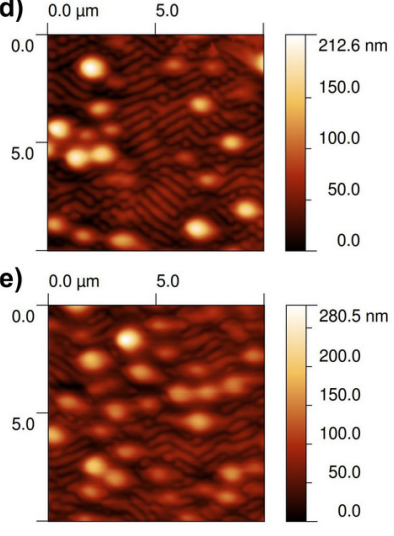


This is significantly larger than both the radiation pressure $P_{\text {laser }} \approx 10^{-5} \mathrm{~Pa}$ exerted by the laser beam during the experiment, and the pressure $P_{\text {laser }} \approx 50 \mathrm{~Pa}$ that could be exerted by other authors in pulsed laser experiments reported by other authors. ${ }^{28}$ An alternative explanation is offered with the anisotropic photo-induced translation diffusion of azobenzenes. ${ }^{9}$ Azobenzene moieties migrate by diffusion along their long axis when they undergo repeated cis-trans isomery. Under a given area $A$, the rate $k$ of cis-trans isomery in a sample of thickness $h$ submitted to a laser intensity $I$ is:

In which $N_{t}$ is the density of azo moieties in the trans isomer in the glass, $\Phi$ is the quantum efficiency for cis-trans isomery, $\sigma$ is the absorption cross-section of the molecules, $\hbar \omega$ is the photon energy and $\left\langle\cos ^{2}(\theta)>\right.$ is the average orientation of the molecules' transition moment with respect to the laser polarization direction. It was observed that when excited with longitudinally polarized laser (perpendicular to the surface), the azo material protrudes out of the surface under the laser beam. ${ }^{29}$ When the molecules are excited with a uniform laser beam with transverse polarization, the molecules mostly tend to move into the plane, with negligible effect on the surface. However, the orientation average $\left\langle\cos ^{2}(\theta)>\right.$ is strongly affected by photo-induced birefringence which orients the molecules perpendicularly to the laser beam polarization. The molecules then can protrude out of the surface, which explains the initial hill formation. Interestingly, this places photo-induced birefringence as a key parameter in SSRG formation. This provides an explanation to the observation that no SSRGs could form in Azoglass-530 under identical laser exposure conditions (Figure 12). This also provides an explanation to the observation that SSRG formation was faster under circularly polarized light compared to linearly polarized light. Indeed, when excited under linear polarization, molecules tend to align into the plane perpendicular to the polarization direction, of which only half is perpendicular to the surface; meanwhile, when excited under circular polarization, molecules tend to align in the direction perpendicular to the polarization plane, which is the direction perpendicular to the surface. This reasoning thus explains why the SSRG formation process happens twice as fast under circularly polarized light compared to linearly polarized light. Looking more carefully at Eqn.(3), birefringence also explains why linear polarization excitation induces a pair of gratings with crossed orientations in fig. $2 \mathrm{~h}$. Indeed, birefringence depletes the population of molecules aligned along the laser polarization. In consequence, the excitation rate $k$ is maximized into a cone of directions centered on the laser polarization, which projects on the surface into the two $\approx \pm 40^{\circ}$ directions observed in figure $2 \mathrm{~h}$.

\section{Conclusions}

Spontaneous surface relief grating formation under singlebeam exposure in general is attributed to the interference pattern between the incident beam with beams scattered by surface defects at the grazing angle. In this study, SSRG nucleation and growth was monitored on samples with various ranges and sizes of surface defects. Formation and propagation of SSRG under linear and circular light irradiation have been studied. On topologically flat surfaces, irradiation initially induces the formation of protrusions with a $500 \mathrm{~nm}$ diameter as a result of mass transport due to repeated cis-trans isomerization. Ripples then gradually form around these defects, leading to the formation of gratings which spread over the sample. For linear polarization, a patchwork of grating domains in two different directions formed, whereas for circular polarization, grains arrange in a circular pattern. It has been proven that light coupling due to the defects, which are capable of diffracting light into the sample, is responsible for SSRG formation. Further, samples with different range of defects were prepared using either corona poling at different temperatures or by spin-coating with various mixtures of solvents. Pore defects do not diffract light at the free surface of the sample and do contribute to the SSRG formation, reinforcing the hypothesis that SSRG formation is a surface-driven process. Optimal growth rate is achieved for defects with dimensions close to the grating pitch, and larger defects slow down SSRG formation. These large defects eventually flatten and elongate in the direction parallel to the light polarization due to directional photofluidization. SSRG nucleation and growth also have been studied on two analogous azobenzene glass-forming derivatives, Azoglass-470 and Azoglass-530. While irradiation of Azoglass-470 resulted in SSRG formation at the same rate as DR1-glass, the gratings saturated at a lower height, and photo-induced surface defects were significantly larger, which is believed to be a consequence of more erratic mass movement due to the presence of two azo groups. On the other hand, no surface deformations, and consequently no gratings, were observed upon irradiation of Azoglass-530. These findings have highlighted some crucial elements of the nucleation of SSRGs that were instrumental in establishing a comprehensive model for their formation.

\section{Acknowledgements}

The authors would like to thank the Canadian Defense Academy Academic Research Program (OL) and the National Science Research Council of Canada Discovery Grants (RGPIN-2015-05743 for RGS and RGPIN-2015-05485 for JMN) for funding, and Dr. Hirohito Umezawa (Fukushima College, Japan) for help with generating electrically poled samples.

\section{Notes and references}

\$ Marvin was used for drawing, displaying and characterizing chemical structures, substructures and reactions, Marvin 15.1.12, 2015, ChemAxon (www.chemaxon.com). 
1. Natansohn, A.; Rochon, P. Photoinduced motions in azocontaining polymers. Chem. Rev. 2002, 102, 4139-4175.

2. Viswanathan, N.K.; Balasubramanian, S.; Li, L.; Tripathy, S.K.; Kumar; J. A detailed investigation of the polarizationdependent surface-relief-grating formation process on azo polymer films. Jpn. J. Appl. Phys. 1999, 38, 5928.

3. Ambrosio, A.; Marrucci, L.; Borbone, F.; Roviello, A.; Maddalena, P. Light-induced spiral mass transport in azopolymer films under vortex-beam illumination. Nat. Commun. 2012, 3, 989.

4. Karageorgiev, P.; Neher, D.; Schulz, B.; Stiller, B.; Pietsch, U.; Giersig, M.; Brehmer, L. From anisotropic photofluidity towards nanomanipulation in the optical near-field., Nat. Mater. 2005, 4,699.

5. Bian, S.P.; Williams, J.M.; Kim, D.Y.; Li, L.A.; Balasubramanian, S.; Kumar, J.; Tripathy, S. Single laser beam-induced surface deformation on azobenzene polymer films. J. Appl. Phys. 1999, 86, 4498-4508.

6. Barrett, C.J.; Natansohn A.L.; Rochon, P.L. Mechanism of optically inscribed high efficiency diffraction gratings in azo polymer films. Phys. Chem. 1996, 100, 8836.

7. Pedersen, T.G.; Johansen, P.M.; Holmes, N.C.R.; Ramanujam, P.S. Mean-field theory of photoinduced formation of surface reliefs in side-chain azobenzene polymers. Phys. Rev. Lett. 1998, 80.1-89.

8. Kumar, J.; Li, L.; Jiang, X.L.; Kim, D.Y.; Lee, T.S.; Tripathy, $\mathrm{S}$. Gradient force: The mechanism for surface relief grating formation in azobenzene functionalized polymers. Appl. Phys. Lett. 1998, 72, 2096.

9. Lefin, P.; Fiorini, C.; Nunzi, J.-M. Anisotropy of the photoinduced translation diffusion of azobenzene dyes in polymer matrices. Pure Appl. Opt. 1998, 7, 71.

10. Ambrosio, A.; Maddalena, P.; Marrucci, L. Molecular Model for Light-Driven Spiral Mass Transport in Azopolymer Films. Phys. Rev. Lett. 2013, 110, 146102.

11. Ahmadi Kandjani, S.; Barille, R.; Dabos-Seignon, S.; Nunzi, J.-M.; Ortyl, E.; Kucharski, S. Multistate polarization addressing using a single beam in an azo polymer film. Opt. Lett. 2005, 30, 1986.

12. Hubert, C.; Fiorini-Debuisschert, C.; Rocha, L.; Raimond, P.;Nunzi, J.-M. Spontaneous photoinduced patterning of azo-dye polymer films: the facts. J. Opt. Soc. Am. B $\mathbf{2 0 0 7}$, 24,1839-1846.

13. Siegman, A.E.; Fauchet, P.M. Stimulated Wood's Anomalies on Laser-Illuminated Surfaces. IEEE J. Quantum Electron. 1986, 22, 1384-1403.

14. Leblond, H.;Barille, R.;Ahmadi-Kandjani, S.; Nunzi, J.-M.; Ortyl, E.; Kucharski, S. Spontaneous formation of optically induced surface relief gratings. J.Phys. B: At. Mol. Opt.Phys. 2009, 42, 205401.

15. Ilnytskyi, J.M.; Neher, D.; Saphiannikova. M. Opposite photo-induced deformations in azobenzene-containing polymers with different molecular architecture: molecular dynamics study. J. Chem. Phys. 2011, 135, 14476.

16. Ishow, E.; Camacho-Aguilera, R.; Guerin, J.; Brosseau, A.; Nakatani, K. Spontaneous formation of complex periodic superstructures under high interferential illumination of small-molecule-based photochromic materials. Adv. Funct. Mater. 2009, 19, 796-804.

17. Teboul, V.; Barille, R.; Tajalli, P.; Ahmadi-Kandjani, S.; Tajalli, H.; Zielinska, S.; Ortyl, E. Light mediated emergence of surface patterns in azopolymers at low temperatures. Soft Matter 2015, 11, 6444-6449.

18. Teboul, V.; Saiddine, M.; Nunzi, J.-M. An isomerizationinduced dynamic heterogeneity in a supercooled glassformer. Phys. Rev. Lett. 2009, 103, 265701.
19. Kirby, R.; Sabat, R.G.; Nunzi, J.-M.; Lebel, O. Disperse and disordered: a mexylaminotriazine-substituted azobenzene derivative with superior glass and surface relief grating formation. J. Mater. Chem. C 2014, 2, 841-847.

20. Mazaheri, L.; Bobbara, S.R.; Lebel, O.; Nunzi, J.-M. Photoinduction of spontaneous surface reliefgratings on Azo DR1 glass Opt. Lett. 2016, 41, 2958-2961.

21. Csete, M.; Hild, S.; Plettl, A.; Ziemann, P.; Bor, Zs.; Marti, $O$. The role of original surface roughness in laser-induced periodic surface structure formation process on polycarbonate films. Thin Sol. Films 2004, 453-454, 114-120.

22. Melito, E.; Laventure, A.; Aldea-Nunzi, G.; Pellerin, C.; Buncel, E.; Lebel, O.; Nunzi, J.-M. Water-triggered spontaneous surface patterning in thin films of mexylaminotriazine molecular glasses. J. Mater. Chem. C 2015, 3, 4729-4736.

23. Umezawa, H.; Nunzi, J.-M.; Lebel, O.; Sabat, R.G. Electric-Field-Induced Nanoscale Surface Patterning in Mexylaminotriazine-Functionalized Molecular Glass Derivatives. Langmuir 2016, 32, 5646-5652.

24. www.gwyddion.net

25. Bennani, O.R.; Al-Hujran, T.A.; Nunzi, J.-M.; Sabat, R.G.; Lebel, O. Surface relief grating growth in thin films of mexylaminotriazine-functionalized glass-forming azobenzene derivatives. New J. Chem. 2015, 39, 91629170.

26. Lin, D.C.; Shreiber, D.I.; Dimitriadis, E.K.; Horkay, F. Spherical indentation of soft matter beyond the Hertzian regime: numerical and experimental validation of hyperelastic models. Biomech. Model Mechanobiol. 2009, 8, 345-358.

27. Plante, A.; Lebel, O.; Soldera, A. Unpublished results.

28. Baldus, O.; Leopold, A.; Hagen, R.; Bieringer, T.; Zilker, S.J. Surface relief gratings generated by pulsed holography: A simple way to polymer nanostructures without isomerizing side-chains. J. Chem. Phys. 2001, 114, 13441349.

29. Ishitobi, H.; Nakamura, I.; Kobayashi, T.; Hayazawa, N.; Sekkat, Z.; Kawata, S.; Inouye, Y. Nanomovement of Azo Polymers Induced by Longitudinal Fields. ACS Photonics 2014, 1, 190-197. 